\title{
Lectura clínica de una sentencia judicial: el derecho a la vida y su aplicación en la toma de decisiones clínicas
}

\author{
AZUCENA COUCEIRO V. ${ }^{1}$ \\ 1. Profesora de Teoría de la Medicina y Humanidades Médicas. Departamento de Especialidades Médico Quirúrgicas. \\ Facultad de Medicina-UPV/EHU-España. \\ Profesora Visitante, Universidad de Concepción (Chile), Universidad Austral (Valdivia-Chile) y Universidad de Magallanes \\ (Punta Arenas-Chile).
}

\section{ABSTRACT \\ Clinical interpretation of a court ruling: the right to life and its application in clinical decision making}

In 2009 , the case of a child affected by acute lymphoblastic leukemia took place. He is treated with good results resulting in complete remission, but relapse occurred later. The parents did not consent to the new treatment, creating a conflict that reaches the legal world and leads to two court rulings of great interest to health professionals. One explained the meaning of the right to life in the clinical practice, and the other dealt with the decision-making process regarding minors based on the theory of the mature minor doctrine, a jurisprudential milestone in Chile. In order to help clarify the meaning of the ruling of the Corte de Apelaciones of Valdivia, its content is presented in clinical terms and ended with the most significant contributions.

(Key words: Bioethics, right to life, limitation of therapeutic effort, informed consent, rejection treatment, mature minor doctrine, minors).

Rev Chil Pediatr 2013; 84 (2): 205-217

\section{RESUMEN}

En el año 2009 se conoce el caso de un niño afectado de leucemia aguda linfoblástica. Es tratado con buen resultado y se obtiene la remisión completa, pero más tarde aparece una recidiva. Los padres no otorgan su consentimiento para el tratamiento, generando un conflicto que llega al mundo judicial y da lugar a dos fallos de gran interés para los profesionales de la salud. Por una parte se señala qué signfica en la práctica clínica el derecho a la vida. Por otra, se desarrolla el proceso de toma de decisiones sobre los menores con base en la teoría del menor maduro, un hito jurispridencial en Chile. Con el propósito de contribuir a esclarecer el significado de la sentencia de la Corte de Apelaciones de Valdivia, se expone su contenido en términos clínicos y se concluye con las aportaciones más significativas.

(Palabras clave: Bioética, derecho a la vida, limitación del esfuerzo terapéutico, consentimiento informado, rechazo de tratamiento, teoría del menor maduro, menores).

Rev Chil Pediatr 2013; 84 (2): 205-217

Recibido el 9 de agosto de 2012, aceptado para publicación el 03 de diciembre de 2012.

Conflictos de interés: La autora declara no tener ningún conflicto de interés en la publicación de este artículo.

Este trabajo cumple con los requisitos sobre consentimiento /asentimiento informado, comité de ética, financiamiento, estudios animales y sobre la ausencia de conflictos de intereses según corresponda. 


\section{Introducción}

En el año 2009 salta a la luz pública el caso de un niño chileno aquejado de una leucemia linfoblástica aguda (LLA) desde el 2005. Cuatro años más tarde sufre una recidiva de su patología. Su madre, tras ser informada sobre la necesidad de completar el estudio e iniciar a la brevedad tratamiento según protocolo de recaídas de la LLA, rechaza el tratamiento de acuerdo con el padre y solicita el alta del niño. Además informa a los profesionales de que va a solicitar la evaluación del menor por un médico naturista, tras la cual comunicará su decisión definitiva al equipo médico.

Como la madre no regresa al hospital ni se pone en contacto con los profesionales de la salud, éstos solicitan al Juzgado de Familia medida de protección, que es concedida en primera instancia por el Tribunal de Familia de Valdivia. Este Tribunal obliga a imponer tratamiento al niño, decisión que más tarde es revocada por la Corte de Apelaciones de Valdivia ${ }^{1}$.

La sentencia de la Corte de Apelaciones marca un hito jurisprudencial en Chile, no tanto en materia de rechazo de tratamiento, del que también se ocupa con detenimiento, cuanto respecto a la forma en que se deben de tomar las decisiones sobre los menores en el ámbito clínico. De aquí la importancia de que los profesionales de la Medicina conozcan su contenido y su aplicación en cualquier escenario clínico.

Si bien esta sentencia ha sido analizada en el ámbito jurídico ${ }^{2}$, no ha ocurrido lo mismo en el ámbito de la Medicina. De aquí el sentido de este artículo, en el que se expondrá el caso, el contenido de la sentencia y las consecuencias que de ella se derivan sobre los elementos que tienen que considerar los profesionales para tomar decisiones clínicas.

\section{Medicina y derecho: el juicio clínico y la sentencia judicial}

Las sociedades modernas se diferencian de las antiguas en que renuncian al uso de la fuerza para resolver los conflictos entre los ciudadanos. Las leyes, pactadas mediante procedimientos participativos y democráticos por todos los ciudadanos, y el mundo judicial que las aplica en los casos conflictivos, tienen de este modo una función de "arbitraje social" en las sociedades que se denominan plurales, en las que las interpretaciones sobre lo que es correcto en una situación determinada son muy diversas.

Una sentencia judicial es, en cierto modo, similar a un juicio clínico, diagnóstico y/ o terapéutico, sobre un caso de cierta complejidad. Esto es así porque tanto el Derecho como la Medicina son disciplinas aplicadas, que deben manejar dos niveles, el teórico y el práctico. De nada vale conocer el contenido de los Principios de Medicina Interna del Harrison si no se sabe aplicar a un caso concreto, lo que requiere un método, la historia clínica, que permita obtener y ponderar las condiciones particulares de cada caso, es decir, los datos clínicos de cada paciente. Del mismo modo, tiene poco sentido conocer el corpus legislativo de un Estado de Derecho si al mismo tiempo no se aplicase a situaciones particulares, a problemas concretos.

Conviene realizar varias presiones precisiones. La primera es señalar que en ninguno de los dos ámbitos la aplicación de la teoría al caso es automática. Requiere contextualizar, ponderar con prudencia las circunstancias del caso, conocer aplicaciones similares -jurisprudencia en el Derecho; casuística en la Medicina-, y llegar así a un juicio que, como mínimo, tenga un buen fundamento. Al igual que en los casos complejos los profesionales de la Medicina pueden llegar a juicios divergentes, las sentencias judiciales también pueden serlo. Eso es lo que ocurrió en las dos sentencias emanadas a raíz de este caso, la del Juzgado de Familia de Valdivia y la de la Corte de Apelaciones de Valdivia, lo que ya es un indicador de la complejidad del mismo.

Es importante comprender las razones y el fundamento de esta divergencia, de la misma manera que interesaría a un profesional de la Medicina saber por qué un mismo caso clínico puede ser diagnosticado y/o tratado de diferente manera por diversos profesionales. Dentro del margen que otorga la variabilidad clíni- 
ca, tal divergencia nos llevaría a reconocer la complejidad del caso y, con toda probabilidad, a estudiar en una sesión clínica especial los diferentes argumentos de los profesionales. Esto es, justamente, lo que se desarrolla en este artículo respecto de las dos sentencias judiciales.

Otra importante precisión conceptual se refiere al papel que tiene la jurisprudencia en la interpretación de los derechos constitucionales. El artículo 19,1 de la Constitución Chilena se refiere al derecho a la vida: "La Constitución asegura a todas las personas el derecho a la vida y a la integridad física y psíquica". Es decir la Constitución afirma que en Chile, como en cualquier otro Estado Constitucional de Derecho, la vida es un valor a proteger reconocido como derecho. Pero a partir de ahí se requiere un trabajo interpretativo sobre cómo hacerlo en cada circunstancia, y eso sólo se puede llevar a cabo conforme aparecen nuevos supuestos sociales, como es el caso de unos padres que rechazan un tratamiento médico para su hijo al tiempo que los profesionales consideran que el niño debe recibirlo.

Esta necesidad de interpretación es aún más evidente en el ámbito sanitario, ya que en las últimas décadas se han producido cambios tecnológicos y culturales que obligan a interpretar, en un contexto de posibilidades técnicas hasta ahora inéditas, lo que significa el derecho a la vida. La tecnología, y más aún la de soporte vital, no debe ser aplicada a los pacientes sin evaluar riesgos y beneficios. La lex artis, sinónimo jurídico de práctica clínica correcta, obliga a evaluar y ponderar el uso de la tecnología ante cada paciente para no caer en malapraxis.

A ello hay que añadir otro cambio reciente, el descubrimiento de los derechos de los pacientes $^{3,4}$. Más allá de la teoría, respetar estos derechos exige que se les informe sobre su situación clínica, y también que se les acompañe en el proceso de toma de decisiones, lo que incluye la posibilidad de que rechacen el tratamiento propuesto. Por tanto, no basta ya con apelar al derecho a la vida para saber con precisión cómo actuar en determinados casos, y es ahí donde la jurisprudencia juega un papel interpretativo fundamental en el ámbito del Derecho.

En resumen, las relaciones humanas conlle- van una toma continua de decisiones. Cuando en un Estado de Derecho hay un conflicto entre las partes que éstas no resuelven por sí solas, se da entrada al mundo judicial.

Se presupone que estos conflictos son controvertidos y novedosos en el ámbito en el que se producen, y de aquí que requieran una interpretación no exenta de complejidad.

Una sentencia judicial como la que es objeto de este artículo es, a efectos prácticos, de la máxima importancia para los profesionales de la Medicina, ya que interpreta el significado del derecho a la vida y especifica los criterios que se deben cumplir para poder forzar la imposición de un tratamiento médico. En palabras de la propia sentencia: "La tarea de la Corte es decidir cuál es el margen constitucional y legal para que el Estado pueda forzar la práctica de un tratamiento médico, y cuáles son los límites que el Estado no debe traspasar en esta materia".

A esto hay que añadir que por tratarse de un caso de un paciente menor de edad, la sentencia aplica lo que se conoce como teoría del menor maduro, que rige la toma de decisiones sobre menores y adolescentes, marcando un hito jurisprudencial en esta materia.

\section{Caso clínico}

El paciente nació en 1997. A los 8 años de edad debutó con mialgias, dolores óseos, decaimiento progresivo y fiebre de $39^{\circ} \mathrm{C}$, por lo que fue ingresado en el servicio de Pediatría del Hospital de Punta Arenas, ciudad en la que residía. Se inicia tratamiento con cloranfenicol por sospecha de fiebre tifoidea. La ausencia de respuesta clínica y la presencia de alteraciones hematológicas -anemia, descenso del recuento de neutrófilos-, conduce a la sospecha diagnóstica de leucemia aguda, por lo que se deriva al niño al hospital de Valdivia en septiembre de 2005.

En este centro se confirma el diagnóstico de Leucemia aguda, tipificada como de estirpe $\mathrm{B}$ (FAB L1). Inmunofenotipo CD10 (+) $80 \%$, HDLA-DR $(+) 50 \%$, sin compromiso del SNC. Se inicia tratamiento en el grupo de riesgo intermedio, por edad, según protocolo PINDA 2002. La madre firma el consentimien- 
to informado. Se procede a instalar un catéter venoso central y se inicia la quimioterapia con buena respuesta, ya que en el día 15 la médula no tiene blastos, y el mielograma del día 33 tampoco muestra blastos.

Durante el tratamiento el niño manifiesta una conducta que hace sospechar una depresión reactiva, cuadro que se asocia a alucinaciones visuales, que se atribuyen a un efecto secundario del uso de corticoides. Es remitido para evaluación psiquiátrica, al tiempo que recibe apoyo con psicoterapia. El 28 de octubre se informa la remisión medular, por lo que es dado de alta.

Hasta diciembre de 2006 ingresa en múltiples ocasiones, bien para recibir pulsos de quimioterapia, o para tratar complicaciones secundarias a la depresión medular producida por la terapia antineoplásica. Una vez completadas las fases intensivas de quimioterapia se inicia, en enero de 2007, protocolo de seguimiento, que requiere un control mensual hasta septiembre de ese mismo año.

La madre solicita autorización para viajar a Punta Arenas, que es denegada por la necesidad del control mensual hasta septiembre de 2007. Dado que el hospital de origen no financia el viaje mensual de la madre y el niño hasta Valdivia, el equipo terapéutico pacta un control intermedio por el pediatra de Punta Arenas, con un viaje cada dos meses a Valdivia. En marzo de 2007 madre e hijo no acuden al control. La primera visita del niño tiene lugar en agosto de 2007, siendo citados de nuevo al mes siguiente para finalizar el tratamiento. En octubre se realizan nuevos exámenes. No se encuentran alteraciones y se señala fin del tratamiento, pues se considera en remisión completa. Se mantiene control periódico en Punta Arenas y en Valdivia, sin que haya evidencias de recidiva.

En marzo de 2009 se detecta un aumento de volumen testicular, por lo que es inmediatamente derivado a Valdivia con sospecha diagnóstica de recidiva testicular, que es confirmada mediante biopsia. Se realiza estudio de extensión y se confirma por histopatología "testículo infiltrado por leucemia linfoblástica CD-10 $(+)^{\text {" }}$, por lo que se oferta tratamiento quirúrgico -orquidectomía- y biopsia en testículo contralateral.
Se informa a la madre de la recaída, de la necesidad de completar el estudio y de iniciar el protocolo de tratamiento de recaídas. Esta rechaza todo nuevo examen y procedimiento diagnóstico, y exige el alta para solicitar la evaluación por un médico naturista, tras la cual comunicará su decisión al equipo tratante. El padre del niño, también informado, respalda la decisión de la madre.

Como no acuden a la cita prevista el mes de marzo, el equipo tratante solicita al Juzgado de Familia de Valdivia una medida de protección, medida que es concedida, fallando ese juzgado a favor de la obligatoriedad del tratamiento. La madre recurre a la sentencia en la Corte de Apelaciones de Valdivia, instancia judicial que revocará el fallo previo.

El niño fallece en el hospital de Punta Arenas el 19 de mayo de 2010, sin que haya habido contacto posterior de los profesionales del hospital de Valdivia ni con él, ni con la familia.

\section{Fallos judiciales: contenido y análisis}

Como ya se ha visto en el relato de los hechos, hay dos instancias judiciales implicadas en este caso, que emiten fallos no coincidentes. Todo comienza cuando el profesional tratante, cuyo paciente es menor de edad, acude al Juzgado de Familia de Valdivia y pide un recurso de protección que le permita tratar al niño en contra de la voluntad de los padres. El fallo de este Juzgado obliga al niño a someterse al tratamiento, argumentando que la protección de la vida y la integridad del niño debe darse en toda circunstancia y sin excepción (tabla 1).

Dice la sentencia: "Si existe tratamiento susceptible de ofrecer alguna posibilidad de sobrevida debe aplicarse hasta agotar los recursos médicos y humanos disponibles". Esta obligación es, a juicio del Juzgado de Familia, absoluta, a diferencia de lo que más tarde afirmará la Corte de Apelación de Valdivia. No es extraña esta divergencia, que puede ser explicarse por varias razones:

- Los Juzgados de Familia ejercen una acción garantista en la protección de los menores, dado que constituyen la parte más desprotegida dentro del ámbito familiar. 
Tabla 1. Sentencias del Tribunal de Familia y de la Corte de Apelación de Valdivia.

Comparación de los fallos judiciales

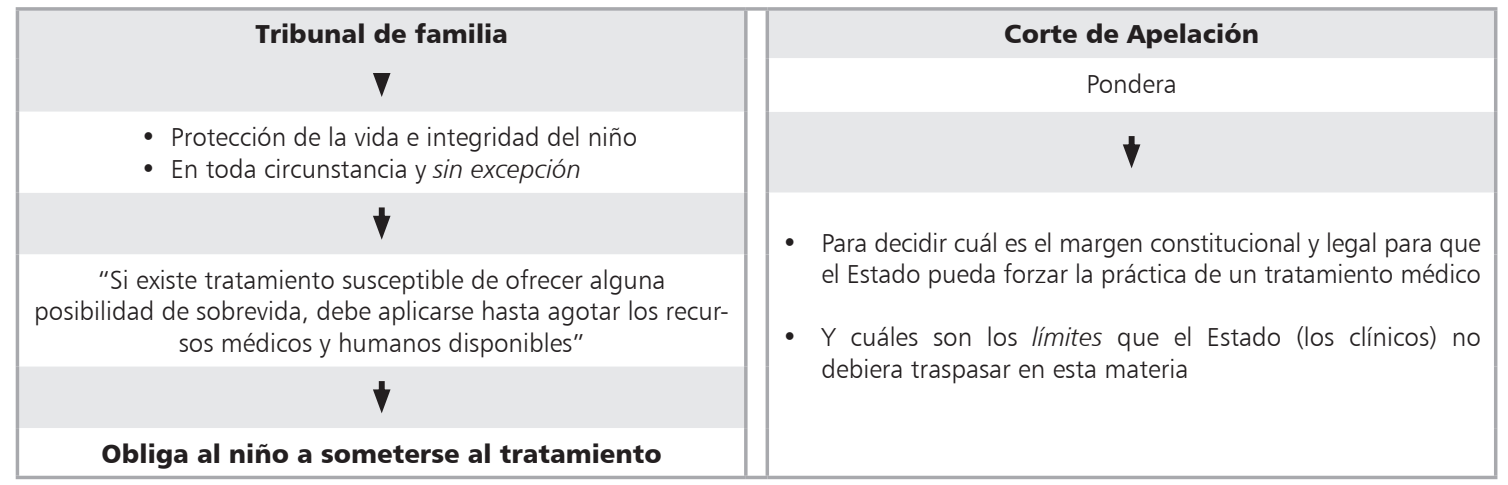

- En general, asumen la posición tradicional prevalente del derecho a la vida en el catálogo de derechos, y la subsiguiente interpretación del derecho a la vida como derecho superior a los restantes.

- Su ámbito de toma de decisiones se circunscribía, hasta ahora, a conflictos relativos a separaciones de los padres, posible maltrato familiar, lesión de los derechos de los menores (por ejemplo, derecho a la escolarización), etc.

- Para los Juzgados de Familia es novedosa la aparición del espacio clínico y de los problemas relacionados con la enfermedad y el tratamiento de un menor. Si hasta ahora ni tan siquiera los adultos podían tomar la decisión de rechazar un tratamiento, rechazo que suele ser el origen de conflictos con los profesionales sanitarios, difícilmente podría plantearse esta situación sobre un menor.

Todo lo anterior puede explicar el que este juzgado obligue en su fallo a que el niño se someta a cualquier tratamiento que ofrezca posibilidades de supervivencia, y que no realice ningún tipo de valoración sobre los riesgos a los que se somete el paciente-efectos secundarios, intervenciones quirúrgicas, internamiento hospitalario- o los beneficios esperados. En cambio, la Corte de Apelaciones efectuará una ponderación de los valores y derechos en conflicto para acotar qué es lo que corresponde hacer. De aquí que se diga en la sentencia que la
Corte trata de fundamentar "cuál es el margen constitucional y legal para que el Estado pueda forzar la práctica de un tratamiento médico y cuáles son los límites que el Estado [léase, los profesionales] no debiera traspasar en esta materia".

Esto es así porque, como también explica la sentencia, no existen obligaciones absolutas. Cuando se produce un conflicto entre derechos constitucionales, en este caso entre el derecho a la vida y a la libertad de elección, hay que llevar a cabo un ejercicio de ponderación ${ }^{5}$.

"El derecho a la vida -continúa la sentencia- es la estructura de plausabilidad sobre la que pueden ejercerse el resto de los derechos, pero eso no significa que prevalezca siempre $y$ sin excepciones". Por tanto, se trata de delimitar cuándo, en qué condiciones y con qué criterios hay que evaluar una situación clínica para tomar la decisión de priorizar la vida -imposición de tratamiento- o la libertad de rechazar el mismo.

Esto es lo que hace la sentencia. En primer lugar delimita cuál es el significado del derecho a la vida y del derecho a la integridad física, pues el segundo es el que ha fundamentado en todos los países el derecho al consentimiento informado y el derecho al rechazo de tratamientos. Y como el caso que suscita su actuación es el de un menor de edad, en el siguiente paso la Corte señala criterios específicos a tener en cuenta en el caso de los menores (tabla 2). 
Tabla 2. Estructura de la sentencia de la Corte de Valdivia

\section{Corte de Apelaciones de Valdivia}

[Apelación de medida de protección, 14 de mayo de 2009]

Significado del derecho a la vida

Deliberación sobre el caso

\section{El derecho a la vida en la sentencia de la Corte de Apelaciones y sus implicaciones clínicas}

Tras el relato de los hechos, la sentencia analiza la jurisprudencia previa del Tribunal Superior de Justicia sobre si está justificado forzar tratamientos médicos no deseados por los pacientes. Para responder a esta pregunta se basa, en primer lugar, en la indicación clínica, que se identifica tras una cuidadosa ponderación de riesgos y beneficios, cuyo equilibrio es lo que permite identificar una praxis como "indicada". En el campo de la Oncología se requiere, específicamente, conocer la probabilidad de éxito del tratamiento y de recuperación del paciente -supervivencia-, y valorar tanto los efectos secundarios como su repercusión sobre su calidad de vida.

Ahora bien, ya no basta con establecer una indicación. Por muy justificada que esté, por muy ajustada que sea a la mejor y más actuali- zada medicina basada en pruebas, no se puede llevar a efecto cuando un paciente bien informado, que comprende la información recibida, las alternativas terapéuticas y sus consecuencias, y que toma una decisión sin coacciones ni presiones indebidas, rechaza el tratamiento ${ }^{6}$. De esta forma, el segundo criterio señalado en la sentencia es la aceptación del paciente que, en el marco de este proceso y cumpliendo con todos sus elementos, toma una decisión sobre sí mismo y sobre su integridad física ${ }^{7,8}$.

En consecuencia, para llevar a cabo un tratamiento se requiere la aceptación por un paciente que cumpla con las condiciones del proceso de consentimiento informado, proceso que no es lo mismo que la mera firma de un formulario ${ }^{9-11}$, como así lo señala la ley de derechos de los pacientes recientemente aprobada en Chile ${ }^{12}$. La expresión "forzar un tratamiento" deja ya de tener sentido cuando hablamos de sujetos adultos y capaces.

Una vez que se ha delimitado de esta forma el derecho a la vida y a la integridad física (tabla 3), las consecuencias para la toma de decisiones en el ámbito clínico que señala esta sentencia son las siguientes:

- "El derecho a la vida no puede entenderse pura y simplemente como la mera continuación de las funciones biológicas". El uso de la tecnología en los enfermos críticos ${ }^{13}$, o de cualquier tratamiento de los enfermos crónicos en fase avanzada, tiene que tener en cuenta además de la cantidad de vida, el pronóstico del paciente. Esto incluye elementos

Tabla 3. Fundamentos del alcance del derecho a la vida en la sentencia de la Corte de Apelaciones de Valdivia

El derecho a la vida en el ámbito de los tratamientos médicos

- El derecho a la vida es condición de plausibilidad sobre la que ejercer el resto de los derechos

- Eso no significa que prevalezca siempre y sin excepciones

Elementos que se requieren para aplicar un tratamiento

- Criterios que justifican forzar tratamientos médicos (obtenidos de la jurisprudencia previa del TSJ):

1. La actuación del profesional no supone un grave atentado contra la calidad de vida del paciente

2. Garantiza en alto grado (\%) la recuperación del paciente

- Cuando la decisión del rechazo es libre, informada y voluntaria, resulta constitucionalmente merecedora de respeto y protección estatal. No se puede intervenir forzando un tratamiento

Conclusiones

- El derecho a la vida no puede entenderse pura y simplemente como la mera continuación de las funciones biológicas

- El derecho a la vida significa:

1. El derecho a conservar la vida

2. El derecho a "hacer la vida", es decir, a elegir los parámetros con los cuales viviremos nuestra existencia física y espiritual

- El Estado no tiene la tarea de imponer, de manera coactiva, siempre y sin excepciones, el deber de vivir 
de calidad de vida tales como efectos secundarios, disminución de las funciones vitales, alteración de las capacidades para realizar actividades de la vida diaria, etc.

- Consecuentemente con el punto anterior, aplicar tratamientos y/o usar la tecnología con el solo objetivo de mantener las funciones biológicas, constituye una mala praxis clínica, con las consecuencias éticas y legales que se derivan de dicha actuación. Para evitarlo es necesario conocer y saber aplicar el concepto clínico de limitación del esfuerzo terapéutico ${ }^{14,15}$, tanto en enfermos críticos como en enfermos crónicos en fase avanzada.

- "Cada ciudadano tiene el derecho a elegir los parámetros con los cuales quiere vivir su existencia física y espiritual'. Ello significa que el derecho a la vida ya no puede ser definido por los profesionales de la salud en virtud de criterios exclusivamente clínicos. Es el paciente quién, en última instancia, decide en el marco de la relación clínica, sobre los elementos de calidad de vida que considera prioritarios en su biografía.

- Por ello, aclara la sentencia, "el derecho a la vida no sólo no puede ser impuesto coactivamente por el Estado" -léase por los profesionales de la salud-, sino que es facultad del ciudadano adulto definir el contenido y alcance de su derecho a la vida en el marco de la salud y la enfermedad ${ }^{16}$.

\section{La toma de decisiones clínicas sobre un menor y la sentencia de la Corte de Apelaciones}

Una vez definido el alcance del derecho a la vida y a la integridad física en el sujeto adulto, la sentencia plantea cómo tomar decisiones sobre los menores. El proceso es el mismo que en el caso de los adultos: primero se establece la indicación y después se establece el proceso de elección, si bien en el caso del menor son los padres quienes, en principio, están facultados para tomar las decisiones sobre el menor bajo su potestad. Ahora bien, esto no significa que los padres puedan tomar cualquier decisión, ni que los menores sean ignorados en el proceso, siendo esta última cuestión una de las aportaciones más interesantes de la sentencia (tabla 4).

En primer lugar, todos los que intervienen

Tabla 4. Proceso de toma de decisiones sobre los menores. Elaboración personal a partir de la Sentencia de la Corte de Apelaciones de Valdivia

El derecho a la vida en la toma de decisiones clínicas sobre un menor

\author{
Procedimiento \\ 1. Acotar la indicación clínica, teniendo en cuenta tanto cantidad como calidad de vida: posibilidad de recuperación de la salud; \\ efectos secundarios de la quimioterapia \\ 2. Los padres toman una decisión \\ 3. Participación del menor en el proceso
}

Discrepancia entre los padres y los profesionales de la salud

- Si se cumplen los criterios que determinan lo que se considera una clara indicación clínica, que pondera riesgos y beneficios -no hay graves efectos secundarios y el tratamiento pronostica la recuperación de la salud en un elevado \%-, y los padres rechazan el tratamiento, se justifica la intervención del Estado

Ámbito de la no maleficencia-no hacer daño protegido por el estado

- Si no se cumplen esos criterios - hay graves efectos secundarios debidos al tratamiento y son bajas las probabilidades de remisión del tumor-, es la familia quien tiene la facultad de decidir, adoptando la decisión que mejor respete sus valores y formas de vida. Ámbito de la beneficencia-hacer el bien respetado por el estado. Decide la familia

Participación del menor en el proceso

- El menor tiene derecho a intervenir en el proceso, a ser escuchado y a participar del mismo (Convención de los derechos del niño; Ley 19.968 de los Tribunales de Familia)

- Su opinión deberá ser tenida en cuenta (será objeto de consideración judicial) en función de varios criterios: edad, capacidad para la comprensión de la situación y capacidad para la toma de una decisión argumentada. Es lo que se conoce como teoría del menor maduro

- Es significativa, para su consideración, la experiencia previa que el menor ha tenido del tratamiento, cómo la percibe y cómo afrontaría una nueva intervención clínica 
en este proceso, los padres y también los profesionales, están obligados por lo que se denomina el criterio del mayor beneficio o "mejor interés" del menor. Su contenido, lo que esto signifique en cada caso, sólo puede ser definido tras ponderar cuidadosamente todos los elementos que permiten sentar una indicación. En el caso de este niño, afectado de una enfermedad oncológica, prestando especial atención al pronóstico, a los efectos adversos de la quimioterapia, y a los efectos secundarios producidos por antibióticos, antihistamínicos y corticoides sobre su calidad de vida.

Para definir cuál es el mejor interés de este niño hay que comenzar por identificar los diversos escenarios clínicos que se suceden. El primero corresponde al primer ingreso, donde tras valoración clínica, diagnóstico de la patología -Leucemia linfobástica aguda, FAB L1, Inmunofenotipo CD10 (+) 80\%, HLA-DR (+) $50 \%-\mathrm{y}$ comprobación de que no hay compromiso inicial del sistema nervioso central, se estima que por la edad del niño pertenece al grupo de riesgo medio. En este grupo el pronóstico es bueno en el $75-80 \%$ de los casos. De hecho se logra la remisión completa.

El segundo escenario ya es diferente. Se trata de una recaída tardía con infiltración testicular. Como la madre no da el consentimiento y los acontecimientos se precipitan, suponemos que no se llega a completar el estudio para saber si la recidiva es extramedular o hay también afectación del SNC. Este dato es fundamental, ya que modifica el pronóstico de ob- tención de una segunda remisión completa del tumor, que pasa del $77 \%$ en el primer supuesto al $49 \%$ en el segundo.

Además del pronóstico, en el segundo escenario es necesario contemplar que además de la orquidectomía y de la biopsia del testículo contralateral, y en función de los resultados del estudio completo, habría que añadir a este tratamiento quimioterapia de mantención, y probablemente pulsos de reinducción según el grupo al que pertenezca el paciente. Tampoco hay que olvidar que, pese a este tratamiento, sólo el 35\% de los pacientes permanece en remisión completa continua. De hecho, en el escrito presentado por el equipo médico al Juzgado de Familia pidiendo un recurso de protección se señala que las posibilidades de sobrevida del paciente se estiman alrededor de un $40 \%$.

Es ahora cuando se puede introducir en el proceso la elección, que en principio corresponde a los padres, tras ser informados con claridad, de manera comprensible y continuada, y manteniendo con ellos una relación clínica de apoyo para afrontar las consecuencias de la toma de decisiones.

¿Hasta dónde pueden decidir los padres? ¿Dónde poner los límites? ¿Cuándo son los padres los únicos legitimados para decidir?. Estas son cuestiones que exigen, siempre y en cada caso, una respuesta prudencial, guiada básicamente por la situación clínica (tabla 5). Con base en este caso se pueden plantear distintos escenarios clínicos:

Tabla 5. Padres y profesionales en la toma de decisiones sobre los menores

\begin{tabular}{|c|c|c|}
\hline Indicación clara & $\begin{array}{l}\text { Incremento de riesgos no tan } \\
\text { claramente indicado }\end{array}$ & Se pierde la indicación clínica \\
\hline $\begin{array}{l}\text { - Adecuada ratio riesgos /beneficios } \\
\text { - Escenario 1: aparición de la patología. } \\
\text { Buen pronóstico } \\
\text { - El mejor interés es tratar al niño } \\
\text { - Si los padres rechazan el tratamiento, } \\
\text { intervención del Estado }\end{array}$ & $\begin{array}{l}\text { - Incremento de los riesgos, pero aún } \\
\text { con pronóstico aceptable } \\
\text { - Escenario } 2 \\
\text { - El mejor interés sigue siendo tratar al } \\
\text { niño } \\
\text { - Negociar con los padres y proporcionar } \\
\text { alternativas }\end{array}$ & $\begin{array}{l}\text { - Inadecuada ratio riesgos/beneficios } \\
\text { - Escenario } 3 \\
\text { - El mejor interés (iniciar un nuevo ciclo } \\
\text { de tratamiento o no iniciarlo), sólo } \\
\text { puede ser definido por los padres }\end{array}$ \\
\hline NO MALEFICENCIA & & BENEFICENCIA \\
\hline EN FUNCIÓN DE SU & DAD: COMUNICACIÓN, ASENTIMIENT & , CONSENTIMIENTO \\
\hline
\end{tabular}


a) Escenario 1: Aparición de la patología. Dado el balance riesgo/beneficio y el buen pronóstico de este paciente, el mejor interés del niño es tratar la enfermedad, intentando minimizar los efectos secundarios colaterales al tratamiento y apoyando psicológicamente tanto a los padres como al menor. Si tras un adecuado proceso de consentimiento los padres se negaran al tratamiento, es el momento de pedir un recurso de protección, teniendo cuidado de no apartar a los padres de la relación clínica, y de explicarles cuanto sea necesario por qué no se debe respetar una decisión que lesiona el mejor interés del menor.

Utilizando el lenguaje de la Bioética, no tratar al niño sería maleficente, hacer daño al menor. Como la función del Estado es la de proteger del daño a los menores, es exigible su intervención y proveer el tratamiento.

b) Escenario 2: Recidiva extramedular sin compromiso del SNC. En esta situación ya hay un incremento de riesgos respecto de la anterior, si bien hay que considerar que el porcentaje de remisión es aún bastante elevado. Aunque la relación entre riesgos /beneficios es peor que la anterior, todavía hay un buen pronóstico $(75 \%)$. Se puede argumentar que el mejor interés del menor pasa por iniciar el tratamiento. Si los padres negaran su consentimiento, conviene averiguar las razones y proporcionarles alternativas que les ayuden a modificar su postura.

En el caso que nos ocupa es claro, desde el principio, el problema que para una familia de Punta Arenas supone vivir en Valdivia todo el proceso de tratamiento oncológico. Manejar este problema es prioritario para lograr la aceptación del tratamiento. Asimismo, para no perder la confianza de los padres, se puede apoyar la consulta con la medicina alternativa, que se ubica dentro del marco legal en Chile, y asegurarse de que ambos tratamientos no colisionan. Es decir, convertir esa consulta a otro ámbito de la Medicina en la vía que permita generar una mejor alianza con los padres.

c) Escenario 3: Recidiva combinada, medular con SNC o con testículo. En esta situación el pronóstico de remisión ya es mucho más bajo. Es probable que los clínicos, con base en su modus operandi habitual, sigan planteando que hay que tratar al paciente. Sin embargo, hemos cruzado el límite: la ratio riesgos/beneficios es ya mucho más baja, y estas condiciones circunscriben una decisión - dar un ciclo más de tratamiento o no darloque sólo pueden tomar los padres.

Otra de las aportaciones de la sentencia de la Corte de Valdivia es, precisamente, señalar que cuanto mayores son los riesgos y efectos secundarios, y menos claros los beneficios, " $\mathrm{el}$ Estado -léase, los clínicos-debe retroceder, y es la familia, con información suficiente, la que tiene que adoptar la decisión que mejor respete sus valores y formas de vida". En el lenguaje de la Bioética estamos ante una elección de Beneficencia.

La familia es un proyecto de valores, una comunión de ideales de vida. Elige la educación de sus hijos, la transmisión de una determinada fe, de una visión del mundo y de los valores que considera prioritarios dentro de su proyecto vital. Todo eso son decisiones de lo que en el marco familiar se considera bueno, beneficente, y el Estado no puede, ni debe, intervenir sobre ellas mientras no traspasen el límite y se conviertan en maleficentes. En definitiva, ni el Estado ni el médico tienen capacidad para definir la beneficencia del menor, por más que tradicionalmente lo hayan hecho as ${ }^{17}$.

De aquí que sea recomendable evitar afirmaciones generales como la de que, en caso de conflicto con los padres, el pediatra tiene un compromiso prioritario con el niño y su derecho a la vida, pues transmiten la idea de que en caso de conflicto debe primar la opinión del profesional, que sería el único cualificado para defender ese derecho ${ }^{18}$. Si algo se desprende de la sentencia de la Corte de Valdivia, y de todo lo anteriormente explicitado, es que esto no es exactamente así. Además también puede darse la posibilidad de que sean los profesionales los que, en su mejor empeño por tratar y mantener la vida, puedan llegar a situaciones maleficentes para el niño, de obstinación terapéutica, en las que se pierde la ratio riesgos/beneficios que justifica una indicación clínica. Y es que, como se afirmó previamente, el criterio del mejor interés del menor obliga tanto a los profesionales de la Medicina como a los padres.

Pero todavía queda otro elemento a tener 
presente. El menor es el gran ausente de este proceso, que habitualmente se desarrolla de forma exclusiva entre los profesionales y los padres. No es extraño que esto ocurra si tenemos en cuenta que, hasta hace muy poco, ni tan siquiera los adultos podían tomar decisiones sobre su enfermedad, y aceptar o rechazar tratamientos. Como ya se ha señalado, los criterios jurisprudenciales respecto del rechazo de tratamiento han sufrido una variación significativa en el sentido de exigir el consentimiento del paciente, pero este cambio, que ha culminado en la ley de derechos de los pacientes, es muy reciente en Chile.

La sentencia de la Corte de Valdivia señala que es necesario recabar la opinión del menor, y fundamenta esta exigencia en la denominada teoría del menor maduro. Dicha teoría, basada en los trabajos de Kolberg sobre la psicología del razonamiento moral y su desarrollo, plantea que la madurez y capacidad para realizar juicios morales se adquiere progresivamente. El ejercicio del juicio moral es un proceso cognitivo, que en situaciones de conflicto permite reflexionar sobre nuestros valores y experiencias, y que se construye de forma activa en interacción permanente con el medio exterior.

Kolberg propuso seis estadios, agrupados en tres niveles diferentes: preconvencional (hasta los 12 años), convencional (12 a 18 años) y postconvencional. Él mismo afirma que la mayoría de las personas no superan el nivel convencional, es decir, que casi todos decidimos con las capacidades cognitivas que se alcanzan alrededor de los 18 años $^{19}$. La propuesta de Kolberg sigue siendo la clave de apoyo de la psicología moral actual, y permite explicar y fundamentar la evolución del pensamiento jurídico en los últimos años sobre la capacidad de los menores para tomar decisiones. Se ha transitado así desde la psicología del desarrollo moral al Bioderecho, lo que conlleva consecuencias de gran interés práctico ${ }^{20}$.

La primera ha sido la reducción paulatina de la fecha de la mayoría de edad en nuestras sociedades. La segunda, la aparición en el ámbito del Derecho de la teoría del menor maduro, que afirma que los derechos civiles o subjetivos existen en el individuo desde el momento en el que éste es capaz de ejercerlos, lo que con sucede en muchos ámbitos de la vida del menor antes de los 18 años. En palabras de la propia sentencia, "la ley reconoce que el proceso de formación de la conciencia humana es gradual, y por lo mismo exige que la opinión del menor sea objeto de consideración judicial, a la luz de su edad y de sus capacidades intelectuales concretas".

En resumen, esta evolución ha conducido a:

- La generación de la doctrina de los derechos del menor con base en la psicología del desarrollo moral.

- El establecimiento de un punto de inflexión en los 14 años, edad a partir de la cual un menor, si goza de madurez, puede ejercer por sí mismo sus propios derechos humanos, debiendo al menos asentir sobre aquellas decisiones que los afectan.

- La generación de una obligación ética y jurídica de respeto al menor en función de su madurez y capacidad para tomar decisiones en el ejercicio de sus derechos. Esto obliga a introducir el modelo de consentimiento informado también en el ámbito de la Pediatría $^{21}$. Dicho modelo será progresivo, en función de la edad, -introducción del menor en la comunicación, asentimiento, consentimiento-, pero no puede ser obviado.

En definitiva, si el modelo de consentimiento se entiende correctamente, como un proceso comunicativo en el seno de la relación clínica que conduce a consensuar la toma de decisiones y no como la mera firma de un documento, las consecuencias en Pediatría son de gran magnitud. En primer lugar, el menor siempre debe ser introducido en el proceso, estableciendo comunicación directa y personal con él, adecuando la información y la forma de comunicación a su edad. Ser escuchado o ser oído es un derecho de los menores que recoge la Ley 19.968 de Tribunales de Familia, y nadie puede ser escuchado cuando ni tan siquiera se le tiene en cuenta en el proceso de relación.

En segundo lugar, a partir de los 12 años el menor debe asentir, por más que sean los representantes quienes consientan. Asentir es un grado más respecto de ser escuchado, y exige atender a las razones y elecciones de los 
menores en la vivencia de su enfermedad, si bien su opinión no sea determinante de forma definitiva. Entre los 16 a 18 años son muchas las legislaciones que, con base en esta teoría, otorgan la capacidad decisoria - capacidad de consentir- al menor, en este caso adolescente, si se comprueba que comprende la información y que tiene la capacidad y madurez suficiente para tomar una decisión, ya que valora las consecuencias y hace una elección sobre la que puede dar razones ${ }^{22,23}$. El profesional sanitario es el garante ante la sociedad de que se dan las mencionadas condiciones.

Volviendo al caso que nos ocupa, el niño debió de ser introducido en el proceso comunicativo de la relación clínica desde el primer momento, en primera persona y no sólo a través de su madre. Cuando aparece la recidiva ya tiene 12 años, una edad significativa en el desarrollo moral, y además "habiendo vivido antes la experiencia de un tratamiento de quimioterapia, estima esta Corte que, aunque fuera nada más que para una mejor ilustración, debió consultarse su parecer".

\section{La deliberación del caso}

Si en los apartados anteriores se han desarrollado los elementos y criterios que fundamentan las decisiones sobre los menores, todavía resta un elemento importante, tanto para el Derecho como para la Ética y la Medicina: la deliberación.

Deliberar significa identificar hechos y datos, identificar valores en conflicto, analizar circunstancias y consecuencias previsibles, y así ponderar y tomar decisiones prudentes que podamos sustentar con argumentos ${ }^{24}$.

No se puede deliberar si no se conocen los hechos -situación clínica del paciente, pronóstico con y sin tratamiento, alternativas a ese tratamiento-; si no se identifican los valores en conflicto; si no se tiene claridad en los conceptos que permiten ponderar entre principios, construir una taxonomía y establecer reglas de prioridad $^{25}$. Lo contrario es una mera opinión subjetiva, carente de valor.

Deliberar también significa que se puede encontrar más de un curso de acción que pueda ser justificado, y que la elección del que se considera el mejor en cada momento, para cada paciente, cada familia, cada menor y cada situación clínica, es un juicio prudencial de ponderación. En concordancia con esto, hay dos aspectos de la sentencia que merece la pena destacar, pues son ejercicio práctico de deliberación sobre este caso.

La primera se refiere a la cuidadosa ponderación que realiza de las actuaciones de la madre para poder concluir que "la respuesta adoptada por la madre, en conjunto con su hijo, es razonada y fundada", pues:

- Tiene como antecedente un tratamiento previo con ciclo de quimioterapia, que además de los efectos secundarios físicos y psicológicos, no fue concluyente, ya que la remisión duró 18 meses.

- Sigue buscando alternativas de tratamiento, en este caso en la naturopatía y alopatía, sin los efectos colaterales nocivos de la medicina tradicional. Es decir, no abandona la búsqueda de lo que ella considera que puede ser bueno para su hijo, al tiempo que no le produce daños.

El segundo aspecto se refiere a la elección de los cursos de acción. Que la madre haya actuado de forma razonable y con fundamento, y que el niño se encuentre en un escenario clínico que definimos previamente dentro del ámbito de la beneficencia, en la que el Estado debe retroceder para que la familia decida, no significa que los profesionales no tengan ya ninguna otra obligación. La sentencia señala que "se debe tratar de ubicar a la madre, cerciorarse una vez más de que comprende la situación actual, y explicarle que, pese a su rechazo inicial, puede tomar otra decisión".

En consecuencia, no debe romperse la relación clínica por el rechazo de tratamiento. Al contrario, los padres, sin ser coaccionados, deben sentir que los profesionales siguen a su lado y mantienen la relación clínica, ya sea respetando la decisión (escenario 3), ya imponiendo tratamiento al niño (escenario 1 ), sin olvidar la necesidad de apoyo de unos padres cuyo hijo está recibiendo tratamiento en contra de su voluntad.

Además hay que añadir que desde el mo- 
mento en el que ya no se plantea tratamiento activo con intención curativa, el equipo de salud tiene la obligación de iniciar la vía del abordaje paliativo, y de establecer el canal institucional necesario para dar entrada a los profesionales del ámbito de los cuidados paliativos. Desde que en el año 2002 se estableció el Consenso Nacional de los protocolos de tratamiento del dolor y control de síntomas para niños con cáncer en etapa avanzada, se han organizado equipos multidisciplinares en cada uno de los 13 centros PINDA del país, encargados de la atención integral de estos pacientes $^{26}$.

El niño y sus padres tienen que ser acompañados en el proceso de muerte que sobrevendrá en meses, con un buen control de síntomas y soporte emocional para afrontar la experiencia. Las obligaciones profesionales no terminan cuando el paciente y sus representantes rechazan el tratamiento, sino que se modifican en el sentido de pasar del tratamiento activo al soporte paliativo.

\section{Conclusiones}

Si algo muestra este caso es que ya no basta con apelar al derecho a la vida para tomar decisiones en la clínica. Primero porque su aplicación requiere ponderar riesgos y beneficios, y adecuar los tratamientos y el uso de la tecnología en función de esa ponderación. Segundo, porque el artículo 19 de la Constitución chilena se refiere "a la vida y a la integridad fisica", que no puede ser invadida sin el consentimiento de los pacientes adultos y capaces, tal y como refrenda la jurisprudencia y la ley de derechos de los pacientes.

En el caso de los menores, es necesario introducir el proceso de consentimiento en la toma de decisiones, adecuado a su edad y a su madurez, y saber delimitar la forma en que padres y profesionales se limitan mutuamente. En este sentido, la sentencia comentada marca un hito en Chile, y debería ser aplicada en el ámbito de la Pediatría, lo que requiere manejar un nuevo modelo de relación clínica también en este ámbito, cuyo contenido teórico y habilidades prácticas debería aprenderse en las Facultades de Medicina ${ }^{27-29}$.

\section{Agradecimientos}

Al doctor Gustavo Cea, Hemato-oncólogo pediatra del Hospital Regional de Valdivia, responsable del PINDA, por su inestimable colaboración en aclarar los datos del caso.

\section{Referencias}

1.- Corte de Apelaciones de Valdivia: Sentencia Autos Rol 5321-2009 (2009), 14 de mayo de 2009.

2.- Vivanco A: Negativa de un menor de edad y de su familia a que este reciba una terapia desproporcionada o con pocas garantías de efectividad. Apelación de medida de protección otorgada por la jueza de Familia de Valdivia. Sentencia de la Ilustre Corte de Apelaciones de Valdivia, de 14 de mayo de 2009. Rev Chil Derecho 2009; 36 : 399-440.

3.- Hernando P: Los derechos de los pacientes, una cuestión de calidad. Rev Calidad Asistencial 2005; 20: 353 6.

4.- Couceiro A, Heredia F: Los derechos de los pacientes y el proyecto de ley en Chile. Vida Médica 2007; 59: 66-73.

5.- Atienza M: Argumentación jurídica. En: Garzón Valdés E, Laporta F (editores). La justicia y el derecho. Madrid: Trotta, 1996: 231-8.

6.- Comité de Bioética de Catalunya: Recomendaciones del Comité de Bioética de Catalunya ante el rechazo de los enfermos al tratamiento. Barcelona, 2010.

7.- Couceiro A: La relación clínica en las sociedades democráticas Bioética y Debat 200; 4: 17-21.

8.- Couceiro A: La relación clínica: historia, modelos, instrumentos y retos. En: Beca JP, Astete C (editores). Bioética clínica. Santiago de Chile: Mediterráneo, 2012; p. 145-59.

9.- Pantoja L: El consentimiento informado: ¿sólo un requisito legal?. Rev Esp Reumatol 2004; 31: 475-8.

10.- Simon P: Diez mitos en torno al consentimiento informado. An Sist Sanit Navar 2006; 29 (supl 3): 29-40.

11.- Pérez C, Jalil R, Canals C, Soto S, Pinto J, Bedregal P, Godoy D: Relación médico-paciente: estudio comparativo entre dos servicios de medicina interna. Rev Med Chile 1998; 116: 1239-45.

12.- Gobierno de Chile: Ley n ${ }^{\circ} 20.584$ Regula los derechos y deberes que tienen las personas en relación con acciones vinculadas a su atención en salud. 13 de abril de 2012.

13.- Monzón JL, Saralegui I, Abizanda R, et al: Recomendaciones de Tratamiento al final de la vida del paciente crítico. Med Intensiva 2008; 32: 121-33. 
14.- Canteros J, Lefeubre O, Toro M, Herrera C: Limitación del esfuerzo terapéutico. Rev Chil Med Intensiva 2007; 22: 93-6.

15.- Irribarren S, Latorre K, Muñoz T, et al: Limitación del esfuerzo terapéutico tras el ingreso en una unidad de Medicina Intensiva. Análisis de factores asociados. Med Intensiva 2007; 31: 68-72.

16.- Chueca Rodríguez $R$ : Los derechos fundamentales a la vida y a la integridad física: el poder de disposición sobre el final de la vida propia. Derecho y Salud 2008; 16: 1-14.

17. Gracia D: Bioética y Pediatría. En: M de los Reyes y M Sánchez (editores). Bioética y Pediatría. Madrid: Ergón, 2010; 29-38.

18.- Mcnab ME, Beca JP: ¿Existen límites en la decisión de los padres sobre el tratamiento de sus hijos?. Rev Chil Pediatr 2010; 81: 536-40.

19.- Kolberg L: Psicología del desarrollo moral. Bilbao: Desclée de Bouwer, 1992.

20.- Simón P, Barrio I: Estadios y evolución de la conciencia moral para tomar decisiones sobre la propia salud: de la Bioética al Bioderecho. En: M de los Reyes y M Sánchez (editores). Bioética y Pediatría. Madrid: Ergón, 2010: 39-48.

21.- Simón P, Barrio I: La capacidad de los menores para to- mar decisiones sanitarias: un problema ético y jurídico. Rev Esp Pediatría 1997; 53: 107-18.

22.- Gracía D, Jarabo Y, Martín N, Ríos J: Toma de decisiones con el paciente menor de edad. Med Clin (Barc.) 2001; 117: 179-90.

23.- Esquerda M, Pifarre J, Gabaldón S: Evaluación de la competencia para tomar decisiones sobre su propia salud en pacientes menores de edad. FMC 2009; 16: 547-53.

24.- Gracia D: La deliberación moral: el método de la ética clínica. Med Clin (Barc) 2001; 117: 18-23.

25.- Atienza M: Juridificar la Bioética: una propuesta metodológica. En: Atienza M (editor). Bioética, Derecho y Argumentación. Bogotá: Palestra, 2004: 38-79.

26.- Minsal: Protocolo. Alivio del dolor y cuidados paliativos para el niño con cáncer, 2005.

27.- Couceiro A, Muñoz M: La enseñanza de la Bioética en Medicina. Una propuesta de desarrollo curricular. Rev Educ Cienc de la Salud 2007; 4: 92-9.

28.- Couceiro A: Cinco mitos sobre la enseñanza de la Bioética en las Facultades de Medicina. Rev Educ Cienc de la Salud 2009; 6: 68-74.

29.- Couceiro A: La enseñanza práctica de la Bioética: diseño curricular, objetivos y materiales docentes. Rev Educ Cienc de la Salud 2012; 9 (2): 69-80. 